\title{
A MIXED PROBLEM OF ACTIVE AEROSOL POLLUTION
}

\author{
HOANG DINH DUNG \\ Institute of Mathematics, NCST of Vietnam
}

\begin{abstract}
The mixed problem of active aerosol pollution is stated in the mathematical modeling of environment problems [1] and was studied by some authors (see [1], [2], $[3], \ldots$ ). In this work we give the solution using the theory of Schwart distributions.
\end{abstract}

\section{Introduction}

Let $G$ be a cylindrical region in the space $R^{3}$ of the point $x=\left(x_{1}, x_{2}, x_{3}\right)$ with sufficiently smooth boundary $\partial G=\partial G_{0} \cup \partial G_{c} \cup \partial G_{h}$, where $\partial G_{0}, \partial G_{c}$ and $\partial G_{h}$ being the bedding $\left(x_{3}=0\right)$, lateral and upper $\left(x_{3}=h\right)$ surfaces, respectively.

Assume that the stack of a plant emits a mixture of pollutants at the point source $x_{0}=\left(x_{1}^{0}, x_{2}^{0}, h^{0}\right) \in G$. Denote by $\varphi_{j}, j=\overline{1, N}$, the concentrations of aerosols. Let these pollutants be converted from one form to another, that is, we may have the following chains:

$$
\varphi_{j}=\varphi_{j 1} \rightarrow \varphi_{j 2} \rightarrow \varphi_{j 3} \rightarrow \ldots, \quad j=\overline{1, N}
$$

For simplicity of presentation, let us consider the conversion process for only a pollutant corresponding to the concentration $\varphi_{1}$ :

$$
\varphi_{1} \rightarrow \varphi_{2} \rightarrow \cdots \rightarrow \varphi_{m}
$$

the $m$-th pollutant is not converted to another form. Then, by $[1], \varphi_{i}, i=\overline{1, m}$, satisfy the following equations and conditions:

$$
\begin{aligned}
& A \varphi_{1}+\sigma_{1} \varphi_{1}=f \\
& A \varphi_{2}+\sigma_{2} \varphi_{2}-\hat{\sigma}_{1} \varphi_{1}=0 \\
& \cdots \ldots \ldots \ldots \ldots \ldots \ldots \\
& A \varphi_{m-1}+\sigma_{m-1} \varphi_{m-1}-\hat{\sigma}_{m-2} \varphi_{m-2}=0 \\
& A \varphi_{m}+\hat{\sigma}_{m-1} \varphi_{m-1}=0
\end{aligned}
$$




$$
\begin{aligned}
& \varphi_{i}(x, t)=\varphi_{i}^{0}(x) \text { for } t=0, x \in G, i=\overline{1, m} \\
& \varphi_{i}=\varphi_{i}^{c} \text { on } \partial \Omega_{c} \text { if } V_{n}=\mathbf{V} \cdot \mathbf{n}<0 \\
& \frac{\partial \varphi_{i}}{\partial x_{3}}=0 \text { on } \partial \Omega_{h} \text { if } V_{n} \geq 0 \\
& \frac{\partial \varphi_{i}}{\partial x_{3}}+\beta \varphi_{i}=0 \text { on } \partial \Omega_{0} \text { if } V_{n} \geq 0
\end{aligned}
$$

where

$$
\begin{gathered}
A=\frac{\partial}{\partial t}+\mathbf{V} \cdot \nabla-\mu \frac{\partial^{2}}{\partial x^{2}}-\mu \frac{\partial}{\partial y^{2}}-\frac{\partial}{\partial y^{2}}-\frac{\partial}{\partial z} \nu \frac{\partial}{\partial z} \\
\operatorname{dic} \mathbf{V}=0
\end{gathered}
$$

$\mathbf{V}=(u, v, w)$ is the wind velocity; $\mu$ and $\nu$ are the horizontal and vertical diffusion coefficients, respectively; $\sigma_{i}, i=\overline{1, m-1}$, is the transformation coefficient of the $i$-th pollutant, $\hat{\sigma}_{i}=\sigma_{i}+s_{i}, i=\overline{1, m-1}, s_{i}$ is the conversion coefficient of the $i$-th pollutant to $(i+1)$-the pollutant; $\varphi_{i}^{0}$ and $\varphi_{i}^{c}, i=\overline{1, m}$, are the given functions; $\mathbf{n}$ is the outer normal to $\partial G ; 0 \leq \beta(x) \leq \beta_{0}, \beta_{0}$ is a constant; $\partial \Omega_{c} \equiv \partial G_{c} \times(0, T)$, $\partial \Omega_{0} \equiv \partial G_{0} \times(0, T), \partial \Omega_{h} \equiv \partial G_{h} \times(0, T), \partial \Omega_{0} \cup \partial \Omega_{c} \cup \partial \Omega_{h} \equiv \partial \Omega, \partial \Omega$ is the boundary of $\Omega, \Omega \equiv G \times(0, T)=\{(x, t): x \in G, 0<t<T<\infty\}$.

\section{Exact solution}

Suppose that there are more than three concentrations $(m>3)$ in the chain (1) and that the components $4,5, \ldots$ are not harmful and may be neglected. Thus, we consider the system (1.2) in the following form:

$$
\begin{aligned}
& \frac{\partial \varphi_{1}}{\partial t}+u \frac{\partial \varphi_{1}}{\partial x}+v \frac{\partial \varphi_{1}}{\partial y}+w \frac{\partial \varphi_{1}}{\partial z}-\mu\left(\frac{\partial^{2} \varphi_{1}}{\partial x^{2}}+\frac{\partial^{2} \varphi_{1}}{\partial y^{2}}\right)-\frac{\partial}{\partial z}\left(\nu \frac{\partial \varphi_{1}}{\partial z}\right)+\sigma_{1} \varphi_{1}=f, \\
& \frac{\partial \varphi_{2}}{\partial t}+u \frac{\partial \varphi_{2}}{\partial x}+v \frac{\partial \varphi_{2}}{\partial y}+w \frac{\partial \varphi_{2}}{\partial z}-\mu\left(\frac{\partial^{2} \varphi_{2}}{\partial x^{2}}+\frac{\partial^{2} \varphi_{2}}{\partial y^{2}}\right)-\frac{\partial}{\partial z}\left(\nu \frac{\partial \varphi_{2}}{\partial z}\right) \\
& +\sigma_{2} \varphi_{2}-\hat{\sigma}_{1} \varphi_{1}=0 \\
& \frac{\partial \varphi_{3}}{\partial t}+u \frac{\partial \varphi_{3}}{\partial x}+v \frac{\partial \varphi_{3}}{\partial y}+w \frac{\partial \varphi_{3}}{\partial z}-\mu\left(\frac{\partial^{2} \varphi_{3}}{\partial x^{2}}+\frac{\partial^{2} \varphi_{3}}{\partial y^{2}}\right)-\frac{\partial}{\partial z}\left(\nu \frac{\partial \varphi_{3}}{\partial z}\right) \\
& +\sigma_{3} \varphi_{3}-\hat{\sigma}_{2} \varphi_{2}=0 \text {. }
\end{aligned}
$$

Let $\sigma_{1} \neq \sigma_{2} \neq \sigma_{3}$ and for the sake of simplicity we assume that $\mu=\nu=$ const $\geq 0$, thus, the last system has the form:

$$
\begin{aligned}
& P \varphi_{1}+\sigma_{1} \varphi_{1}=f, \\
& P \varphi_{2}+\sigma_{2} \varphi_{2}=\hat{\sigma}_{1} \varphi_{1}, \\
& P \varphi_{3}+\sigma_{3} \varphi_{3}=\hat{\sigma}_{2} \varphi_{2},
\end{aligned}
$$


where

$$
P=\frac{\partial}{\partial t}+\mathbf{V} \cdot \nabla-\mu \Delta
$$

With the same way as in [1] (see Sec. 7.3, Chap. 7) one may reduce (2.1) to the following system:

$$
\begin{aligned}
& P \psi_{1}+\sigma_{1} \psi_{1}=f \\
& P \psi_{2}+\sigma_{2} \psi_{2}=\hat{\sigma}_{1} f \\
& P \psi_{3}+\sigma_{3} \psi_{3}=\hat{\sigma}_{1} f
\end{aligned}
$$

where

$$
\begin{aligned}
& \psi_{1}=\varphi_{1}, \quad \psi_{2}=\hat{\sigma}_{1} \varphi_{1}+\left(\sigma_{1}-\sigma_{2}\right) \varphi_{2} \\
& \psi_{3}=\hat{\sigma}_{1} \varphi_{1}+\left(\sigma_{1}-\sigma_{3}\right) \varphi_{2}+\frac{1}{\hat{\sigma}_{2}}\left(\sigma_{1}-\sigma_{3}\right)\left(\sigma_{2}-\sigma_{3}\right) \varphi_{3} .
\end{aligned}
$$

Thus we have three independent problems with the same form and they may be solved by one same method. After solving this problem one can find $\varphi_{i}(i=1,2,3)$ from (2.3). Therefore, it is sufficient to solve one of the equations for $\psi_{i}(i=1,2,3)$.

For an illustration we consider the following problem:

$$
\begin{aligned}
& Q \psi=f \text { in } \Omega \\
& \psi=\psi_{0}(x) \text { for } t=0, x \in G \\
& \psi=g \text { on } \partial \Omega_{c} \text { if } V_{n}<0 \\
& \frac{\partial \psi}{\partial n}=0 \text { on } \partial \Omega_{0} \cup \partial \Omega_{h} \text { if } V_{n} \geq 0
\end{aligned}
$$

where

$$
Q \equiv P+\sigma \text {. }
$$

Note that the existence and uniqueness of the solution for the problem (2.4), (2.5) is proved quite analogously as in [4] for an analogous mixed problem (see Secs. 2, 3).

We now wish to find a solution in the space of Schwartz distributions $D^{\prime} \overline{R_{+}^{-}} \times$ $R^{3}$ ). For this purpose, we first continue the functions $\psi$ and $f$ by zero onto $\Omega^{-} \equiv R^{1} \times E^{3} \backslash \bar{\Omega}$ and denote the extended functions by $\tilde{\Psi}$ and $\tilde{f}$ respectively. In accordance with the general theory (see Sec. 15, Chap.3, [5]) the differential equation corresponding to the problem $(2.4),(2.5)$ in $D^{\prime}\left(\overline{R_{+}^{1}} \times R^{3}\right)$ has the form

$$
Q \tilde{\Psi}=\tilde{f}+\tilde{\Psi}_{0}(x) \times \delta(t)-\frac{\partial}{\partial n}\left(\tilde{g} \delta_{\partial \Omega}\right),
$$

where $\tilde{\Psi}_{0}(x)$ is the extended distribution of $\Psi_{0}(x)$ by zero onto $\overline{G^{-}} \equiv R^{3} \backslash G, \delta(t)$ is the Dirac distribution, $-\frac{\partial}{\partial n}\left(\tilde{g} \delta_{\partial \Omega}\right)$ is the generalized double layer on $\partial \Omega$ with surface moment density $\tilde{g}$ uniquely defined by the function $g$ and the surface $\partial \Omega$. 
Let $\mathbf{V}=$ const, $\sigma=$ const $\geq 0$. Then, the fundamental solution in $D^{\prime}\left(R_{+}^{1} \times R^{3}\right)$ of the operator $Q$ may be written in the form [4]:

$$
E(x, t)=\frac{\theta(t)}{(4 \mu \pi t)^{3 / 2}} \exp \left\{-\left[\sigma t+\frac{|x-\mathbf{V} t|^{2}}{4 \mu t}\right]\right\}
$$

where $\theta(t)$ is the Heaviside unit function. form:

Thus, the unique solution in $D^{\prime}\left(\overline{R_{+}^{1}} \times R^{3}\right)$ of the problem (2.4), (2.5) has the

$$
\tilde{\Psi}=\tilde{f} * E+\left[\tilde{\Psi}_{0}(x) \times \delta(t)\right] * E-\left[\frac{\partial}{\partial n}\left(\tilde{g} \delta_{\partial \Omega}\right)\right] * E .
$$

We now consider the summands of (2.8) in a classical case. Let $f$ be a finite (with respect to $x$ ) distribution on $G, f$ belong to $L^{2}(G)$ and satisfy the following estimate in each region $\{(x, t): x \in G, 0 \leq t \leq T\}$ :

$$
|f(x . t)| \leq C_{t, \varepsilon}(f) e^{\varepsilon|x|^{2}}
$$

where $\varepsilon$ is an arbitrary constant $\geq 0, C_{t, \varepsilon}$ is a constant and it may be assumed that this quantity does not decrease with respect to $T$. Then the dispersion potential $\tilde{\psi}_{1} \equiv \tilde{f} * E$ exists and is expressed by the form:

$$
\tilde{\Psi}_{1}=\int_{0}^{t} \int_{R^{3}} \frac{\tilde{f}(y, \tau)}{[4 \mu \pi(t-\tau)]^{3 / 2}} \exp \left\{-\left[\sigma(t-\tau)+\frac{|(x-y)-\mathbf{V}(t-\tau)|^{2}}{4 \mu(t-\tau)}\right]\right\} d y d \tau
$$

It is easy to verify that $\tilde{\psi}_{1}$ satisfies the following estimate:

$$
\left|\tilde{\Psi}_{1}(x, t)\right| \leq \frac{C_{t, \varepsilon}(f) e^{2 \varepsilon|x|^{2}}}{(1-16 \varepsilon \mu t)^{3 / 2}} e^{4 \varepsilon|\mathrm{V}|^{2} t^{2}} \cdot t \quad \text { for } 0<t<\frac{1}{16 \varepsilon \mu} .
$$

From the last inequality follows, for arbitrary $A>0$,

$$
\tilde{\Psi}_{1}(x, t) \underset{t \rightarrow+0}{\stackrel{|x|<A}{\longrightarrow} 0}
$$

Consider the surface dispersive potential $\tilde{\Psi}_{2} \equiv\left[\tilde{\Psi}_{0}(x) \times \delta(t)\right] * E$. Assuming that $\psi_{0}(x) \in L^{2}(G)$, we have

$$
\tilde{\Psi}_{2}(x, t)=\frac{\theta(t) e^{-\sigma t}}{(4 \pi \mu t)^{3 / 2}} \int_{R^{3}} \tilde{\Psi}_{0}(y) e^{-\frac{\left|x-y-V_{t}\right|^{2}}{4 \mu t}} d y
$$


Using the equality:

$$
\int_{R^{3}} e^{-|\zeta|^{2}} d \zeta=\pi^{3 / 2}
$$

from (2.11) we get

$$
\lim _{t \rightarrow+0} \tilde{\Psi}_{2}(x, t)=\Psi_{0}(x), \quad x \in G
$$

The third summand $\tilde{\Psi}_{3} \equiv-\frac{\partial}{\partial n}\left(\tilde{g} \delta_{\partial \Omega}\right) * E$ in (2.8) is a potential of double layer on $\partial \Omega$. Let the given function $g$ be continuous on $\partial \Omega_{c}$, then one has

$$
\tilde{\Psi}_{3}=\int_{0}^{T} \int_{\partial G} \tilde{g}(\zeta, \gamma) \frac{\partial E(x-\zeta, t-\gamma)}{\partial n_{\zeta}} d s_{\zeta} d \gamma
$$

where

$$
\tilde{g} \equiv \begin{cases}g & \text { on } \partial \Omega_{c}, \\ 0 & \text { on } \partial \Omega_{0} \cup \partial \Omega_{h} \\ 0 & \text { for } t \leq 0, x \in \bar{G} .\end{cases}
$$

It follows from (2.5), (2.8) and (2.13) that the given function $g$ must satisfy the following condition:

$$
\int_{0}^{T} \int_{\partial G_{c}} g(\zeta, \gamma) \frac{\partial E(y-\eta, \tau-\gamma)}{\partial \eta_{\zeta}} d s_{\zeta} d \gamma=g(y, \tau) \text { on } \partial \Omega_{c} .
$$

One may reduce $(2.14)$ to the following equation

$$
\int_{0}^{T} \int_{\partial G_{c}} g(y-\zeta, \tau-\gamma) \frac{\partial E(\eta, \gamma)}{\partial \eta_{\zeta}} d s_{\varsigma} d \gamma=g(y, \tau), \quad(y, \tau) \in \partial \Omega_{\mathrm{c}} .
$$

Taking (2.7) into account, we see that the kernel of the last integral equation processes integrable singularity. The investigation for the integral equations with the kernel of similar form is presented in [6] (see 15, Chap. 4). tion

Finally, it is easy to verify that the fundamental solution $E$ satisfies the equa-

$$
Q_{C \ell} E=0 \text { in } \Omega \text {, }
$$

where $Q_{C \ell}$ is the differential operator $Q$ with classical derivatives. Hence, by virtue of $(2.10),(2.12)-(2.16)$ one may verify directly that the function $\tilde{\Psi}$ defined by $(2.8)$ is the classical solution of the problem $(2.4),(2.5)$. Note that this solution is a piecewise continuously differentiable function, for the condition of continuous in $\bar{\Omega}$ solution, instead of the condition (2.15) one has a nonhomogeneous integral equation. 
Thus, we have obtained the following result.

Theorem. The unique solution in $D^{\prime}\left(\overline{R_{+}^{1}} \times R^{3}\right)$ of the mixed problem (2.4), (2.5) is expressed by the form (2.8). The classical solution in the class of continuous differentiable functions for this problem has the form:

$$
\begin{aligned}
\tilde{\Psi}= & \int_{0}^{t} \int_{R^{3}} \frac{\tilde{f}(y, \tau)}{[4 \mu \pi(t-\tau)]^{3 / 2}} \exp \left\{-\left[\sigma(t-\tau)+\frac{|(x-y)-\mathbf{V}(t-\tau)|^{2}}{4 \mu(t-\tau)}\right]\right\} d y d r \\
& +\frac{\theta(t) e^{-\sigma t}}{(4 \pi \mu t)^{3 / 2}} \int_{R^{3}} \tilde{\Psi}_{0}(y) \exp \left\{-\frac{|x-y-\mathbf{V} t|^{2}}{4 \mu t}\right\} d y \\
& +\int_{b}^{T} \int_{\partial G} g(\zeta, \gamma) \frac{\partial E(x-\zeta, t-\gamma)}{\partial n_{\zeta}} d s_{\zeta} d \gamma, \quad\left(x_{\zeta} t\right) \in \Omega,
\end{aligned}
$$

where the function $E(x, t)$ is defined by $(2.7)$.

This work is partially supported by the National Basic Research Program in Natural Sciences, Vietnam.

\section{REFERENCES}

1. Marchuk G. I. Mathematical modelling in environmental problems, Science, Moscow, 1982 (in Russian).

2. Dang Quang A and Nguyen Dong Anh. On numerical modelling for dispersion of active pollutants from an elevated point source, Vietnam J. of Math., No 3, 24 (1996), 315-325.

3. 'Pal D. and Sinha A. K. On a numerical dispersion model for gaseous and particulate pollutant from area source, Inter. Conf. on Tropical micrometeo. and air Pollution, Delhi, India, 1988.

4. Hoang Dinh Dung anf Nguyen Cong Dieu. On the problem of air pollution, Acta Math. Vietnam, No 121 (1996), 27-38.

5. Vladimirov V.S. Generalized functions in Mathematical physics, Mir, Moscow, 1979.

6. Ladijenka O. A. and Solonhicov V. A. Linear and quasi-linear parabolic equations, Science, Moscow, 1967 (in Russian).

Received March 22, 2000

BÀI TOÁN HỖN HỢP CỬA QUÁ TRÌNH Ô NHIẼ̃M KHÍ THẢI CÓ HOẠT TÍNH

Bài toán hỗn hợp của quá trình ô nhiễm khí thải có hoạt tính được phát biểu tổng quát trong [1] và đã được một sổ tác giả quan tâm (xem [1], [2], [3],..). Trong bài này, sử dụng lý thuyết các phân bố (các hàm suy rộng) chúng tôi cho nghiệm tổng quát và nghiệm cổ điển của bài toán này. 